\title{
Sleep And Positive Emotions In Adolescents With Anorexia Nervosa In Relation To The Covid-19 Pandemic
}

\section{Lucia Rossi}

IRRCS Istituto delle Scienze Neurologiche di Bologna

\section{Catarina Da Silva}

Salvator University Hospital

Isabelle Charvin

Salvator University Hospital

David Da Fonseca

Salvator University Hospital

Flora Bat-Pitault ( $\triangle$ Flora.BAT@ap-hm.fr)

Salvator University Hospital https://orcid.org/0000-0002-8306-9307

\section{Research Article}

Keywords: Adolescent, Anorexia nervosa, Covid-19, Sleep alterations, Insomnia, Positive emotions.

Posted Date: January 6th, 2022

DOI: https://doi.org/10.21203/rs.3.rs-1035022/v1

License: (9) This work is licensed under a Creative Commons Attribution 4.0 International License. Read Full License 


\section{Abstract}

Purpose: The recent COVID-19 pandemic, and its consequent lockdown measures raised concerns about people's mental health. Identified vulnerable groups may be especially at risk. This study investigated the impact of this unprecedent situation on sleeping patterns and emotionality in adolescents with anorexia nervosa (AN).

Methods: Variations in sleeping patterns and emotionality of 101 adolescents with $\mathrm{AN}$, assessed during the pandemic (Covid-19 Group), and the year before (No-Covid-19 Group) were analyzed. We further investigated these variations over time: before the lockdown, 9 months later and after the lockdown.

Results: Adolescents in the Covid-19 Group had significantly higher rates of sleep alterations and lower rates of positive emotions, compared to the No-Covid-19 Group. A higher insomnia severity index score and a tendency to lengthen sleep latency time were also associated with the pandemic period. Considering three different groups of patients over time, the kinetics of the impact of the changes shows that the worsening of values is not strictly limited to the acute lockdown phase but is increasing over time.

Conclusions: These sleep and emotionality features could have participated to increase severity and incidence of AN in adolescents during the Covid-19 pandemic. As such, these features could represent therapeutic targets to optimize the care of adolescents with AN, particularly urgent to improve considering the kinetic of the deterioration. Tools such as those provided by cognitive behavioral therapy for insomnia (CBTi) and by the positive psychology seem appropriate to guide clinicians in this context.

Level of evidence: Level V, descriptive study (evaluation data retrospectively studied).

\section{Introduction}

The coronavirus disease 2019 (Covid-19) pandemic involved profound changes in daily life, with consequences on mental and physical health of the worldwide population. The strict lockdown measures, mainly expressed by the "stay home" message while keeping social distancing have shown to significantly influence people's eating habits, exercise behaviors, and body image [1]. The impact of such restrictions imposed on the entire population has been the focus of a large amount of research, however, the study on the consequences on the well-being of children and adolescents is surprisingly scarce [2]. This highly restrictive context may have been particularly delicate for adolescents' lives, most likely affecting their core activities, such as part-time employment, sports participation, and creative deeds. It has been suggested that the consequent loss of meaningful social interactions and activities may have contributed to diminish physical activity, increased negative affect, increased lethargy or napping, along with greater consumption of screen time and online social networking [3]. Although there is still no data available on the long-term impact of this crisis, the Covid-19 pandemic seem to have ranging impacts on youth development and mental health, with increased psychiatric disorders, such as Post-Traumatic Stress, Depressive, and Anxiety Disorders, as well as grief-related symptoms [2]. A recent review indeed 
show that that may be case, and that Covid-19 is particularly associated with depression and anxiety in adolescent cohorts [4].

Children and adolescents with preexisting mental health difficulties such as anxiety, depression, and those with neurodevelopmental disorders, may be especially at risk [5], as suggested by studies describing an increase in symptoms severity [6], [7]. Furthermore, this trend tends to increase as enforced isolation continues [8].

There is however, less evidence regarding eating disorders (ED), even this particular context raised increasingly concerns about ED. Indeed, a recent epidemiologic study showed that during 2020 showed, the incidence of ED was $15.3 \%$ higher compared with previous years. Furthermore, a higher proportion of ED patients had suicidal ideation or attempted suicide[9]. In line with a scenario of adverse effects, a pilot study [10] found that after 2 weeks of quarantine, nearly $40 \%$ of adults with ED reported a worsening of ED symptomatology and almost $60 \%$, increased anxiety. During the Covid-19 pandemic, patients with AN were at risk for deterioration of ED and general symptoms, with an increase of eating, shape and weight concerns, drive for physical activity, loneliness, sadness, and inner restlessness [11].Moreover the impact on the care system was also quite significant, thereby aggravating the therapeutic access to patients [12].In addition to lower protective capacity and access to care, three different pathways to explain the exacerbation of ED behaviors during the pandemic were proposed [13]: (1) the disruptions and restrictions to daily activities; (2) the greater consumption of social media; and (3) the emotional distress, worry and fear of contagion.

Individuals' sleeping habits, challenged by factors such as reduced exposure to sunlight, diminish physical activity and psychological distress during the pandemic, have undeniably been affected [14]. While studies with adults showed an increase in sleep duration, of sleep phase delay along with a decrease in sleep quality [14], [15], a study conducted with university students, found instead a reduction in sleep duration and a delay in the timing of sleep [ref]. In agreement, another study, showed that during the Covid-19 context, adolescents delayed bed and wake-up times and shifted chronotype towards eveningness. [16].

This alteration in sleeping patterns with the Covid-19 pandemic may be a critical indicator for adolescents' development, mental health and well-being. Sleep duration and quality have been known to promote positive emotions and mood [17]. Indeed, the Covid-19 period was accompanied by important psychological costs: a significant increase in negative emotionality [18], [19] along with a reduction in positive emotions [19].

This link between sleep patterns and negative emotionality is a relevant clinical predictor of AN risk and severity [20]-[23]. Sleeping problems and the consequent increase of negative emotions was found to occur during the pandemic, and may have function as triggers to develop or aggravate cases of AN during this period. 
This recent traumatic event has, undeniably influenced mental health in a non-trivial way. We may begin to grasp few and more obvious effects, but attention need to kept regarding long-term effects. This study aimed at investigating the impact of the Covid-19 context on the link between sleeping patterns and the emergence of negative emotions in AN. To do so, we estimated the variations of sleep and emotionality in a well characterized group of adolescents with $\mathrm{AN}$, before and during the Covid-19 pandemic. We further investigated these variations in sleeping patterns and emotionality over time, by comparing these profiles between three groups of patients: before the confinement, 9 months later and after the lockdown.

\section{Methods}

\subsection{Participants}

Participants in this study took part of a multidisciplinary intensive ED program in the specialized EDs unit of the Salvator University Hospital in Marseille (France), between March 2019 and March 2020 (No-Covid19 group), and between March 2020 and June 2021, till the end of the lockdown in France (Covid-19 Group). Participants meeting criteria for an intake assessment in the program were children and adolescents aged from 11 to 18 years with AN. Every patient assessed during these two time periods ( $N=$ 101) was included in the study, without exclusion criteria. To investigate the impact of the Covid-19 pandemic over time, we further analyzed the kinetics of its influence on sleep and emotional variables, as assessed by questionnaires (see below) in the three following time windows: (a) the year before lockdown (March 2019 - March 2020; N= 50); (b) the first 9 months - between the beginning of the first lockdown and the end of the second lockdown (March to December 2020; $N=31$ ), and (c) in the remaining 6 months until the end of the lockdown months (December 2020 - June 2021; $N=20$ ).

\subsection{Procedures}

The AN diagnostic was established by a child and adolescent psychiatrist specialized in EDs at the first assessment. Diagnosis was based on the criteria of the DSM-5 [24] as well as on the Schedule for Affective Disorders and Schizophrenia for School-Age Children (K-SADS-PL) [25], a widely used semistructured diagnostic interview to assess mental disorders in children and adolescents (6-18 years old). The participants' assessment collected clinical data (sex, age, weight, height and calculated Body Mass Index, BMI), as well as psychometric data. This was accomplished by self-administered questionnaires for all patients.

The Pittsburgh Sleep Quality Index (PSQI) [26] is a self-rated questionnaire which assesses sleep quality and disturbances over a 1- month time interval. Nineteen items generate 7 component scores: subjective sleep quality, sleep latency, sleep duration, habitual sleep efficiency, sleep disturbances, use of sleeping medication and daytime dysfunction. The sum of scores for these seven components yields a global score [26]. Sleep latency, Sleep duration, Bedtime and Wake time were extracted from the raw data of PSQI for each participant. The Insomnia Sleep Index (ISI) [27]. The ISI assess the severity of insomnia in a 7-items scale, rated on a 5-point scale (ranging from $0=$ not at all interfering, to $4=$ very much interfering). The total score ranges from 0 to 28 (with higher scores indicative of more severe insomnia). 
[27]. The Emotionality was evaluated with the Positive and Negative Emotionality Scale (EPN-31). The EPN-31 is a self-questionnaire composed of 31 items, corresponding to the 31 basic emotional states. Each item is rated on a 7-point scale (ranging from $1=$ not experienced at all, to $7=$ experienced this affect several times each day). Three main scores are calculated: positive, negative and surprise affects [28]. Depressive symptoms were assessed with the Children's Depression Inventory (CDI), a selfreport inventory composed of 27 items, each one consisting of three statements that are graded in severity and are assigned numerical values from 0 (absent or normal behavior for age) to 2 (severe). A total score is obtained by adding the numerical values assigned to each choice marked. The score ranges from 0 to 54, with a pathological threshold of 15 [29]. Anxious symptomatology was assessed with the State-Trait Anxiety Inventory (STAI-Y) [30], an inventory with two separate scales: the STAY Form Y-1 consisting of 20 items measuring the respondent's feeling in that moment, and the STAY Form Y-2 consisting of the next 20 items measuring how the respondent generally feels. All items are rated on a 4point scale (ranging from $1=$ not at all, to $4=$ very much so for the intensity of current feeling, and from 1 = almost never, to $4=$ almost always for the frequency of feeling in general) [28]. For both scales, total scores range from 20 to 80 (total score $\leq 35$ corresponds to very low anxiety and a score $>65$ corresponds to very high anxiety). The Eating Disorder Inventory-2 (EDI-2) [31] is a self-report questionnaire with 11 subscales for measuring attitudes and behaviors related to AN and bulimia nervosa. The 91 items are rated on a 6-point scale: $0=$ never, $1=$ rarely, $2=$ sometimes, $3=$ often, $4=$ usually, and $5=$ always. Each item is score with a scoring system which transforms scores ranging from 0 to 3 rather than 0 to 5 : a score from 1 to 3 is assigned to "symptomatic" response (always $=3$, usually = 2 and often $=1$ ), and 0 is assigned to the three "asymptomatic" responses (sometimes, rarely and never). Subscale scores are obtained by adding all item scores on each subscale. We chose to calculate the EDI2 total score as the sum of the 11 subscales scores to indicate the AN severity. [32].

All adolescents included in this study were volunteers. The experiment adhered to the Declaration of Helsinki and experimental procedures were approved by the Ethics Committee of Aix-Marseille University (approval No. 2020-10-08-001) and the local general data protection regulation. Participants and/or parental informed consent were obtained from all participants at the time of testing.

\subsection{Statistical Analysis}

The statistical analyses were performed using SPSS 20.0 software. We used a non-parametric approach to account for the non-normality of some clinical variable frequencies in the full sample. First, univariate analysis (Mann-Whitney U test) was conducted to compare sleep characteristics, emotionality, anxious and depressive elements, and ED symptoms between the two groups of adolescents evaluated before and after the Covid-19 lockdown (March 17, 2020). Bonferroni corrections were used in the multiple comparisons for the EPN-31 sub-dimensions. Next, Kruskal-Wallis tests and Dunn's test for multiple comparisons were used to compare sleep and emotionality between the three groups: Group a (participants assessed the year before the first lockdown); Group b (participants assessed from the beginning of the first lockdown to the end of second lockdown); and Group c (participants assessed from 
the end of second lockdown - to the end of the lockdown measures in France). All statistical tests were performed bilaterally with an alpha level of $5 \%$, and p-values less than 0.05 were considered significant.

\section{Results}

A total of 101 participants with AN (99 girls (97.0\%) and 3.0\% of boys), aged 11 to 18 years old, with an average age of 14.73 years old $(S D=2.23)$ were included in this study. The mean BMI was $15.13(S D=$ 2.22).

The results showed that sleep disturbances were significantly greater in the Covid-19 Group (adolescents with AN evaluated after the beginning of the first Covid-19 lockdown) than in the No-Covid-19 Group (adolescents with AN evaluated before the first Covid-19 lockdown), in particular regarding insomnia rate and severity (See Table 1). A trend in sleep latency and total sleep quality score was found between the groups (See Table 1). Results also revealed that the No-Covid-19 Group experienced significant more positive emotions than the Covid-19 Group, especially the experience of emotions of happiness and tenderness (See Table 1).Analyses regarding the impact of the Covid-19 pandemic over time, considered the three Groups (A, B, and $C$ ). Results showed that the ISI was significantly greater in the Group $C(M=11.90, S D=6.38, p=0.04)$ than in the Group A $(M=7.57, S D=5.69$, See Figure 1$)$. The Group $C$ also showed to have experiences significantly less positive emotions $(M=38.67, S D=14.62, p=0.01)$ compared to the Group A ( $M=51.05, S D=12.51$, See Figure 2). This was particularly the case of feeling of tenderness (Group C: $M=20.83, S D=7.43$; Group A: $M=27.23, S D=6.61, p=0.01$, See Figure 3).

\section{Discussion}

To our knowledge, this is the first study describing variations of sleeping patterns and emotionality during the Covid-19 pandemic in adolescents with AN. Considering sleeping patterns, findings of the current study revealed increased insomnia severity and a tendency for longer sleep latency and later wake time associated with the pandemic period. This finding is in line with prior research carried out on the adult general population during the quarantine period, mainly showing a decrease of sleep quality [14], [15], [33] and an increase in insomnia rate and severity [34], [35]. Some studies in adults [14] and adolescents [16], [36] also described significant changes in sleep timing, with later bedtime and wake up time, confirming our data. However, contrary to the previously mentioned studies, we did not find an increase in sleep duration in our sample of adolescents with AN. The absence of this protective factor [36] and the finding of increased sleep difficulties in people with higher levels of depression, anxiety and stress symptomatology [14], may instead be related to raising fears of long-term consequences on mental health of adolescents with AN in the course of their disease. Indeed, it has been suggested that sleep alterations, and particularly insomnia, may persist after an acute stress phase and are often precursors of psychiatric disorders and suicide in adults [37], [38]. In adolescents, the increased risk of psychiatric disorders, such as depression, and substance use, has been associated with a worse regulation of risk-taking behaviors, and with potential negative impacts on school performance as well [39], [40]. Other studies suggest that sleep alterations are precursors and severity markers in AN [20]- 
[22]. Although our study did not find a significant augmentation in AN severity during the pandemic, the significantly ascending evolution kinetics of insomnia and the sleep alterations observed throughout this period may raise fears of an increase in the incidence and severity of these adolescents in the coming months. Sleep quality seem to highly relate to emotionality, in particular, poorer sleep is strongly associated to diminish experiencing positive emotions [17], which could explain the increased sleep disturbances found here.

In line with the study Lin and colleagues [34], our findings also revealed, more than an increase in negative emotions, a significant decrease in the experience of positive emotions in adolescents with AN during the pandemic, especially emotions of joy and tenderness. So far, research on Covid-19 pandemic essentially showed an increase in negative emotions [18], [34], while its impact on positive emotions in youth with AN is still limited [41]. The same trend is found in the literature regarding the interaction between sleep and emotionality, with the influence on positive emotions being largely unexplored. Few studies however found sleep duration to be positively associated with happiness, and that lower sleep quality is associated with unhappiness [17], [42].

According to the positive psychology framework, positive emotions such as joy, not only are necessary for managing present challenges such as a pandemic period, but they also build resilience crucial for managing future adversity. Positive psychology suggests that patients may be able to change their behaviors and broaden their perception when experiencing some degree of positive emotion. This effect could have important implications for EDs, as there is experimental evidence suggesting patients have cognitive inflexibility and attention to detail rather than the big picture [43]. Therefore, facilitating positive emotional states and broadening problem-solving skills seem highly relevant to aid individuals with ED [44]. Given the negative impact of the pandemic on these two related clinical variables in AN (sleep and positive emotionality) and on the ascending evolution kinetics of these difficulties throughout this period, knowledge about interventions targeting these aspects and decrease the impact of Covid-19 at a short, medium and long-term, seem fundamental for clinicians [45]-[47].

To reduce sleep alterations, sleep health education and sleep hygiene should be the first priority to prevent long-term adverse outcomes associated with chronic insomnia and mental health problems [48]. Sleep hygiene refers to ensuring bright light exposure during the day but not during the night to enhance sleep-inducing melatonin production, not drinking caffeinated coffee or tea in the evening, reducing alcohol intake, not exercising immediately prior to bedtime, avoiding clock-watching during the night, and ensuring an optimal room and body temperature to fall asleep. For patients with chronic insomnia, the Cognitive and Behavioral Therapy for Insomnia (CBT-I), the first-line treatment for insomnia, should be made more widely available [37], [49]. The key elements of this effective insomnia treatment are sleep hygiene, stimulus control; relaxation interventions; cognitive reappraisal (challenging and replacing dysfunctional ideas about sleep problems and its causes); paradoxical intention (asking individuals to try to stay awake instead of trying to fall asleep), and sleep restriction (restricting the time spent in bed based on the individual's average current nightly sleep duration). Mindfulness, the practice of paying attention to the present moment, purposefully and nonjudgmentally, has been gaining popularity as 
adjunct treatment for adults and adolescents with a range of physical and mental health problems. Mindfulness-based interventions show promising results in various areas including the treatment of sleep disorders in adolescence [50], [51], and CBT-I shows promising effects also for children and adolescents [52].

The Covid-19 pandemic is an unprecedented, traumatic event significantly affecting the mental health of people with ED, possibly through the exacerbation of emotion regulation difficulties. The findings of the current study may further provide clues to a model to guide clinicians in the management of AN patients, suggesting the therapeutic need targeting the antecedents that trigger negative emotions, such as sleep troubles, and increase therapeutic interventions that enhance positive emotions [47], such as reappraisal strategies. The positive psychology frameworks support the development of mental health strategies that focus on promoting competence, resilience, and a sense of efficacy, at both individual and collective levels. Consistent with this, adaptive emotional coping strategies, such as reappraisal, can be an important resilience factor in reducing the negative impact of the pandemic [54]. While shedding light on the link between sleep and emotionality in AN in this particular context, this study presents several limitations. The design did not allow us to confirm the potential causal links between sleep disturbances, emotionality, mental health, and severity of AN in our sample of adolescents. Although using standardized and reliable measures, the lack of more objective measures of sleep would allow us to confirm and specify subjective sleep disturbances. In conclusion, this study confirmed that sleep disturbances are associated to emotionality in adolescents with AN, in particular regarding the Covid-19 pandemic. The impact of these disturbances on global mental health and AN severity, well established in the literature, leads us to emphasize the importance of their management using valid and effective tools. This encourages further research on the effectiveness of therapeutic tools such as CBT-I, mindfulness based-interventions and reappraisal strategies in $\mathrm{AN}$, consistent with the positive psychology framework. This perspective may be successfully incorporated into crisis response and recovery phases of disaster mental health efforts to address the need of adolescents suffering from AN, which seems particularly urgent given the trend confirmed from our data, and which is likely to continue long after the acute phase.

\section{Strength And Limits}

Our study was conducted in a well-defined, homogeneous sample of adolescents with AN diagnosed according to the DSM-5 criteria (APA, 2003). We present for the first time data on sleep and positive emotions in adolescents with AN in relation to the Covid-19 pandemic. This study presents nevertheless some limitations. The design did not allow us to confirm the potential causal links between sleep disturbances, emotionality, mental health, and severity of AN in our sample of adolescents. Although using standardized and reliable measures, the lack of more objective measures of sleep would allow us to confirm and specify subjective sleep disturbances.

\section{What is already known on this subject?}


Research carried out on the adult general population during the quarantine period showed a decrease of sleep quality and an increase in insomnia rate and severity. Some studies have also showed an increase of negative emotions. The reduction of sleep duration and quality could have an emotional impact in adolescence, increasing the experience of negative emotions. Sleep disturbance is often reported by patients with AN, and several studies found that sleep alterations are precursors and severity markers in AN.

\section{What does this study add?}

To our knowledge, this is the first study describing the variations of sleep patterns and emotionality during the Covid-19 pandemic in adolescents with AN. Our findings show shows significantly higher rates of sleep alterations and lower rates of positive emotions in adolescents with AN in the Covid-19 Group compared to No-Covid-19 Group. Moreover, our findings suggest that this impact, not strictly limited to the acute lockdown phase but increasing over time, may provide a model to guide clinicians in the management of AN patients. Our findings further underline the therapeutic need targeting antecedents that trigger negative emotions, such as sleep troubles, and to increase therapeutic interventions that enhance positive emotions.

\section{Declarations}

Funding: not applicable.

Conflicts of interest/Competing interests: the authors have no conflict to declare.

Ethics approval: all procedures performed in this study involving human participants adhered to the Declaration of Helsinki (1964) and its later amendments, and experimental procedures were approved by the Ethics Committee of the Aix-Marseille University (approval No. 2020-10-08-001) and the local general data protection regulation.

Consent to participate/ Consent for publication: all patients included volunteered to participate in this study. At the time of testing, all the participants and their parents gave their informed consent.

Availability of data and material: The datasets generated during and/or analyzed during the current study are available from the corresponding author on reasonable request.

Code availability: All statistical analyses were completed using SPSS 20 software.

\section{References}

1. Robertson M, Duffy F, Newman E, Prieto Bravo C, Ates HH, Sharpe H (2020) "Exploring changes in body image, eating and exercise during the COVID-19 lockdown: A UK survey," Appetite, vol. 159, no. October p. 105062, 2021, doi: 10.1016/j.appet.2020.105062 
2. Guessoum SB, Lachal J, Radjack R et al (2020) "Adolescent psychiatric disorders during the COVID19 pandemic and lockdown," Psychiatry Research, vol. 291. Elsevier Ireland Ltd, p. 113264, Sep. 01, doi: 10.1016/j.psychres.2020.113264

3. Becker SP, Gregory AM (2020) Editorial Perspective: Perils and promise for child and adolescent sleep and associated psychopathology during the COVID-19 pandemic. J Child Psychol Psychiatry 61:757-759. no. 7doi: 10.1111/jcpp.13278

4. Nearchou F, Hennessy E, Flinn C, Niland R, Subramaniam SS (2020) Exploring the impact of covid-19 on mental health outcomes in children and adolescents: A systematic review. Int J Environ Res Public Health 17(22):1-19. doi: 10.3390/ijerph17228479

5. Bell DB, Catherine VTalbot (2020) Exploring the impact of the COVID-19 pandemic and UK lockdown on individuals with experience of eating disorders. 10.1186/s40337-020-00319-y

6. Breaux R, Dvorsky MR, Marsh NP et al (2021) Prospective impact of COVID-19 on mental health functioning in adolescents with and without ADHD: protective role of emotion regulation abilities. $J$ Child Psychol Psychiatry Allied Discip 62(9):1132-1139. doi: 10.1111/jcpp.13382

7. Colizzi M, Sironi E, Antonini F, Ciceri ML, Bovo C, Zoccante L (2020) Psychosocial and behavioral impact of COVID-19 in autism spectrum disorder: An online parent survey. Brain Sci 10(6):1-14. doi: 10.3390/brainsci10060341

8. Loades ME, Chatburn E, Higson-Sweeney N et al (2020) Rapid Systematic Review: The Impact of Social Isolation and Loneliness on the Mental Health of Children and Adolescents in the Context of COVID-19. J Am Acad Child Adolesc Psychiatry 59(11):1218-1239. doi: 10.1016/j.jaac.2020.05.009

9. Taquet M, Geddes JR, Luciano S, Harrison PJ (2021) Incidence and outcomes of eating disorders during the COVID-19 pandemic. Br J Psychiatry 1-3. doi: 10.1192/bjp.2021.105

10. Fernández-Aranda F, Casas M, Claes L et al (2020) COVID-19 and implications for eating disorders. European Eating Disorders Review 28:239-245. no. 3doi: 10.1002/erv.2738

11. Schlegl S, Maier J, Meule A, Voderholzer U (2020) Eating disorders in times of the COVID-19 pandemic-Results from an online survey of patients with anorexia nervosa. Int $J$ Eat Disord 53(11):1791-1800. doi: 10.1002/eat.23374

12. Spettigue W, Obeid N, Erbach $M$ et al "The impact of COVID-19 on adolescents with eating disorders: a cohort study", doi: 10.1186/s40337-021-00419-3

13. Rodgers RF, Lombardo C, Cerolini S et al (2020) The impact of the COVID-19 pandemic on eating disorder risk and symptoms. Int J Eat Disord 53(7):1166-1170. doi: 10.1002/eat.23318

14. Cellini N, Canale N, Mioni G, Costa S (2020) Changes in sleep pattern, sense of time and digital media use during COVID-19 lockdown in Italy. J Sleep Res 29(4). doi: 10.1111/jsr.13074

15. C.Blume MHSchmidt (Jul. 2020) Effects of the COVID-19 lockdown on human sleep and rest-activity rhythms. Curr Biol 30(14):R795-R797. doi: 10.1016/J.CUB.2020.06.021

16. Genta FD, Neto GBR, Sunfeld JPV et al (Jul. 2021) "COVID-19 pandemic impact on sleep habits, chronotype, and health-related quality of life among high school students: a longitudinal study," $J$. Clin. Sleep Med., vol. 17, no. 7, pp. 1371-1377, doi: 10.5664/JCSM.9196 
17. Shen L, van Schie J, Ditchburn G, Brook L, Bei B (2018) Positive and Negative Emotions: Differential Associations with Sleep Duration and Quality in Adolescents. J Youth Adolesc 47(12):2584-2595. doi: 10.1007/s10964-018-0899-1

18. Brooks SK, Webster RK, Smith LE et al (2020) "Rapid Review The psychological impact of quarantine and how to reduce it: rapid review of the evidence," www.thelancet.com, vol. 395, doi: 10.1016/S0140-6736(20)30460-8

19. Li S, Wang Y, Xue J, Zhao N, Zhu T (Mar. 2020) The Impact of COVID-19 Epidemic Declaration on Psychological Consequences: A Study on Active Weibo Users. Int J Environ Res Public Health 17(6). doi: 10.3390/IJERPH17062032

20. Bat-Pitault F, Da Silva C, Charvin I, Fonseca DD (Aug. 2021) Sleep disturbances in anorexia nervosa subtypes in adolescence. Eat Weight Disord 26:1845-1852. doi: 10.1007/s40519-020-01033-z

21. Lombardo C, Ballesio A, Gasparrini G, Cerolini S (2020) “Effects of acute and chronic sleep deprivation on eating behaviour," Clin. Psychol., vol. 24, no. 1, pp. 64-72, Mar. doi: 10.1111/CP.12189

22. Malcolm A, Toh WL (2021) The contribution of sleep to anorexia nervosa severity. Eat Weight Disord. doi: 10.1007/S40519-021-01286-2

23. Puccio et al (May 2016) A systematic review and meta-analysis on the longitudinal relationship between eating pathology and depression. Int J Eat Disord 49(5):439-454. doi: 10.1002/EAT.22506

24. American Psychiatric Association (2013) Diagnostic and statistical manual of mental disorders (5th ed.).

25. Kaufman J, Birmaher B, Brent DA, Ryan ND, Rao U (2000) K-SADS-PL [1]. J Am Acad Child Adolesc Psychiatry 39(10):1208. doi: 10.1097/00004583-200010000-00002

26. Buysse D, Reynolds C, Monk T (1989) The Pittsburgh Sleep Quality Index: a new instrument for psychiatric practice and research. Psychiatry Res 28(2):193-213. doi: 10.1016/01651781(89)90047-4

27. Vallière. BC, Morin A (2001) Validation of the Insomnia Severity Index as an outcome measure for insomnia research. Sleep Med 2(4):297-307. doi: 10.1016/S1389-9457(00)00065-4

28. Pélissolo J, Rolland P-D (2007) "[Dimensional approach of emotion in psychiatry: validation of the Positive and Negative Emotionality scale (EPN-31)]," Encephale., vol. 33, no. 3 Pt 1, pp. 256-263, doi: 10.1016/S0013-7006(07)92037-0

29. K. M., "The Children's Depression, Inventory (CDI).," Psychopharmacol Bull. 21(4)995-8. PMID 4089116,

30. Spielberger JG, Gorsuch C, Lushene RL, Vagg R (1983) "Manual for the State-Trait Anxiety Inventory (Form Y1 - Y2)," Consult. Psychol. Press. Palo Alto, vol. IV,

31. Garner (1991) Eating Disorder Inventory2: Professional manual.

32. Guelfi JD, Criquillion-Doublet S, Divac S, Dardennes R (1995) “Le ‘Eating Disorder Inventory’ (EDI).,” Masson,Paris, pp. 249-260, 
33. Hartley S, Colas des Francs C, Aussert F et al (2020) Les effets de confinement SARS-CoV-2 sur le sommeil: enquête en ligne au cours de la quatrième semaine de confinement. Encephale 46(3):S53S59. doi: 10.1016/j.encep.2020.05.003

34. Lin L, Wang J, Ou-yang X et al (Jan. 2021) "The immediate impact of the 2019 novel coronavirus (COVID-19) outbreak on subjective sleep status," Sleep Med., vol. 77, pp. 348-354, doi: 10.1016/j.sleep.2020.05.018

35. Morin CM, Bjorvatn B, Chung F et al (Nov. 2021) Insomnia, anxiety, and depression during the COVID19 pandemic: an international collaborative study. Sleep Med 87:38-45. doi:

10.1016/j.sleep.2021.07.035

36. Saxvig IW, Pallesen S, Sivertsen B, Hysing M, Evanger LN, Bjorvatn B (2021) "Sleep during COVID-19related school lockdown, a longitudinal study among high school students," J. Sleep Res., Oct. doi: $10.1111 /$ jsr. 13499

37. Morin CM, Carrier J (2021) "The acute effects of the COVID-19 pandemic on insomnia and psychological symptoms," Sleep Medicine, vol. 77. Sleep Med, pp. 346-347, Jan. 01, doi: 10.1016/j.sleep.2020.06.005

38. Sher L "COVID-19, anxiety, sleep disturbances and suicide," Sleep Medicine, vol. 70. Sleep Med, p. 124, Jun. 01, 2020. doi: 10.1016/j.sleep.2020.04.019

39. de Zambotti M, Goldstone A, Colrain IM, Baker FC (2018) "Insomnia disorder in adolescence: Diagnosis, impact, and treatment," Sleep Medicine Reviews, vol. 39. Sleep Med Rev, pp. 12-24, Jun. 01, doi: 10.1016/j.smrv.2017.06.009

40. Bat-Pitault F, Fonseca DD (2012) "Réduction du temps de sommeil chez les adolescents: Conséquences et prise en charge ?," Archives de Pediatrie, vol. 19, no. 10. Arch Pediatr, pp. 10951099, Oct. doi: 10.1016/j.arcped.2012.07.004

41. Tchanturia K, Dapelo MAM, Harrison A, Hambrook D (2014) Why Study Positive Emotions in the Context of Eating Disorders? Curr Psychiatry Rep 17(1):537. doi: 10.1007/s11920-014-0537-x

42. Lee J, Kang J, Rhie S, Chae KY (2013) "Impact of Sleep Duration on Emotional Status in Adolescents.," J. Korean Child Neurol. Soc., vol. 21, no. 3, pp. 100-110, Sep.

43. Wang SB, Gray EK, Coniglio KA et al (2021) Cognitive rigidity and heightened attention to detail occur transdiagnostically in adolescents with eating disorders. Eat Disord 29(4):408-420. doi: 10.1080/10640266.2019.1656470

44. Tchanturia K, Dapelo MAM, Harrison A, Hambrook D "Why Study Positive Emotions in the Context of Eating Disorders?", doi: 10.1007/s11920-014-0537-x

45. Hisler GC, Twenge JM (May 2021) Sleep characteristics of U.S. adults before and during the COVID19 pandemic. Soc Sci Med 276. doi: 10.1016/j.socscimed.2021.113849

46. Monteleone AM (Oct. 2021) The impact of the COVID-19 pandemic on eating disorders: A paradigm for an emotional post-traumatic stress model of eating disorders. Eur Neuropsychopharmacol 51:84-86. doi: 10.1016/J.EURONEURO.2021.05.009 
47. Machado PPP, Pinto-Bastos A, Ramos R et al (Dec. 2020) Impact of COVID-19 lockdown measures on a cohort of eating disorders patients. J Eat Disord 8(1). doi: 10.1186/s40337-020-00340-1

48. Morin G, Carrier, Bastien (2020) Sleep and circadian rhythm in response to the COVID-19 pandemic. 111:654-657. 10.17269/S41997-020-00382-7. 5

49. Altena E, Baglioni C, Espie CA et al (2020) "Dealing with sleep problems during home confinement due to the COVID-19 outbreak: Practical recommendations from a task force of the European CBT-I Academy," Journal of Sleep Research, vol. 29, no. 4. J Sleep Res, Aug. 01, doi: 10.1111/jsr.13052

\section{Table}

Table 1. Comparison of Sleep, Emotionality, anxious and depressive symptoms in the No-Covid-19 and Covid-19 Groups of adolescents with AN. 


\begin{tabular}{|c|c|c|c|}
\hline & & & p-values \\
\hline & $N=50$ & $N=51$ & \\
\hline $\mathrm{Age}^{\mathrm{b}}$ & $14.89 \pm 2.28$ & $14.59 \pm 2.19$ & - \\
\hline $\mathrm{BMI}^{\mathrm{b}}$ & $14.91 \pm 1.60$ & $15.35 \pm 2.67$ & - \\
\hline Sex boys ${ }^{a}$ & $2(4 \%)$ & $1(2 \%)$ & - \\
\hline CDI total score ${ }^{b}$ & $15.92 \pm 9.90$ & $18.44 \pm 9.14$ & - \\
\hline STAIY-state ${ }^{b}$ & $46.91 \pm 15.85$ & $50.59 \pm 13.56$ & - \\
\hline ISI mean score ${ }^{b}$ & $7.57 \pm 5.70$ & $10.49 \pm 6.88$ & 0.037 \\
\hline Clinical insomnia $^{a}$ & $7(14.0 \%)$ & $17(33.3 \%)$ & 0.026 \\
\hline PSQI total score ${ }^{b}$ & $6.05 \pm 3.78$ & $7.71 \pm 4.42$ & 0.062 \\
\hline Sleep latency ${ }^{b}$ & $27.37 \pm 20.65$ & $47.32 \pm 46.25$ & 0.068 \\
\hline Sleep duration ${ }^{b}$ & $8.02 \pm 1.14$ & $7.74 \pm 1.75$ & - \\
\hline Bedtime $^{b}$ (hours) & $20.54 \pm 5.62$ & $19.90 \pm 6.64$ & - \\
\hline Wakeup time ${ }^{\text {b }}$ (hours) & $7.21 \pm 1.05$ & $7.67 \pm 1.29$ & 0.070 \\
\hline \multicolumn{4}{|l|}{ EPN-31 ${ }^{b}$} \\
\hline Positive emotions & $51.05 \pm 12.55$ & $42.38 \pm 14.57$ & 0.004 \\
\hline Negative emotions & $72.39 \pm 27.57$ & $75.48 \pm 25.55$ & - \\
\hline Happiness & $23.85 \pm 7.22$ & $19.79 \pm 7.56$ & 0.011 \\
\hline Tenderness & $27.20 \pm 6.60$ & $23.79 \pm 7.20$ & 0.020 \\
\hline EDI-2 total score & $79.91 \pm 47.25$ & $87.24 \pm 49.73$ & - \\
\hline
\end{tabular}

$\otimes$ Data are presented as number of subjects (\%)

$\otimes$ Data are presented as mean \pm SD

BMI : Body Mass Index, CDI : Children's Depression Inventory, STAIY : State-Trait Anxiety Inventory, ISI : Insomnia Severity Index, PSQI : Pittsburg Sleep Quality Index, EPN-31 : Positive Negative Emotions, EDI-2: Eating Disorder Inventory -2, Clinical insomnia corresponds to an ISI mean score $>14$

\section{Figures}




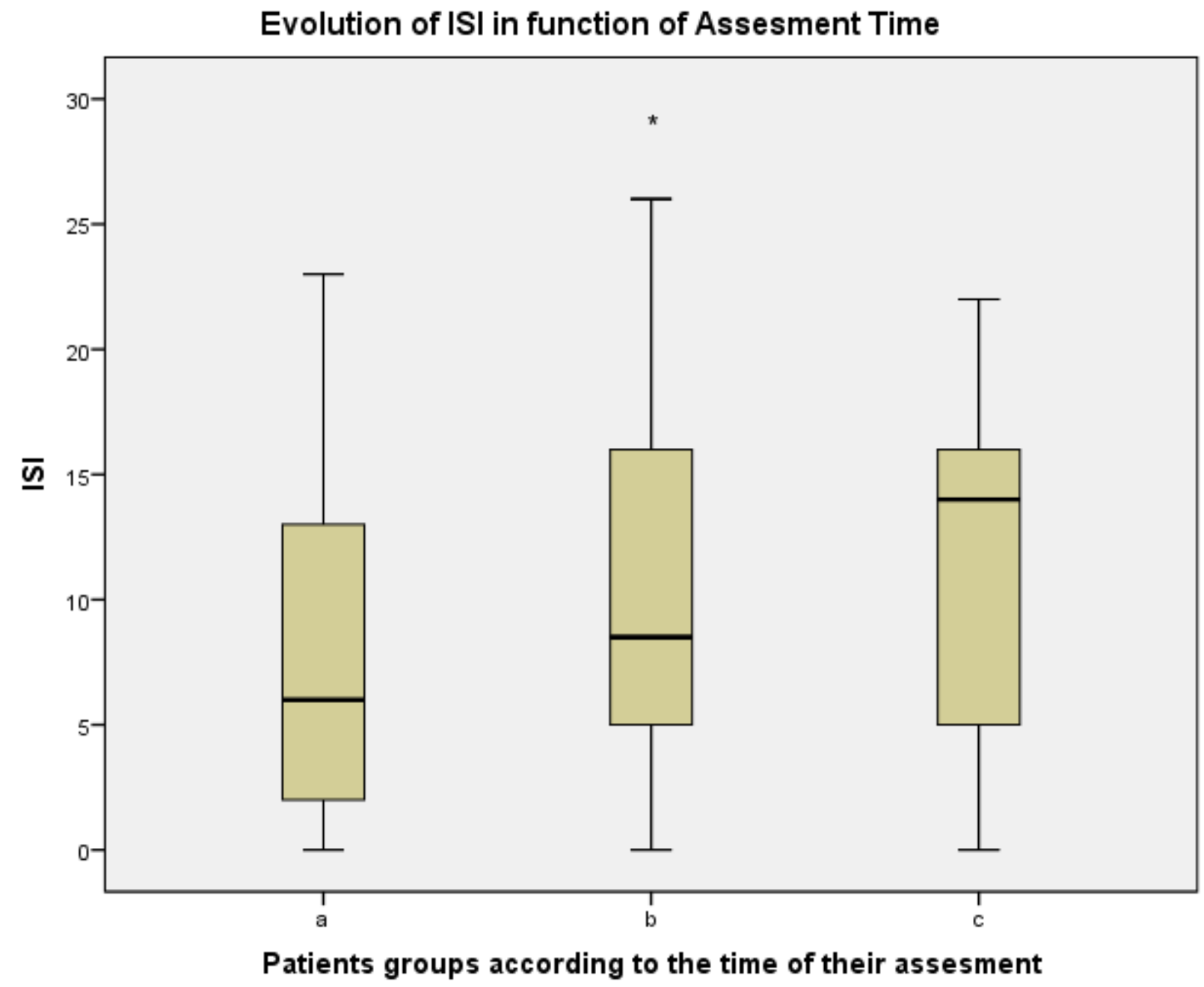

Figure 1

Evolution of ISI score Assessment Time

* $\mathrm{p}<0.05 ;$ ISI: Insomnia Severity Index

Group a: participants assessed the year before the first lockdown (March 17, 2020)

Group b: participants assessed from the beginning of the first lockdown to the end of second lockdown (March 17, 2020 - December 15, 2020)

Group c: participants assessed from the end of second lockdown - to the end of the lockdown measures in France (December 15, 2020 - June 20, 2021) 


\section{Evolution of Positive Emotion in function of Assesment Time}

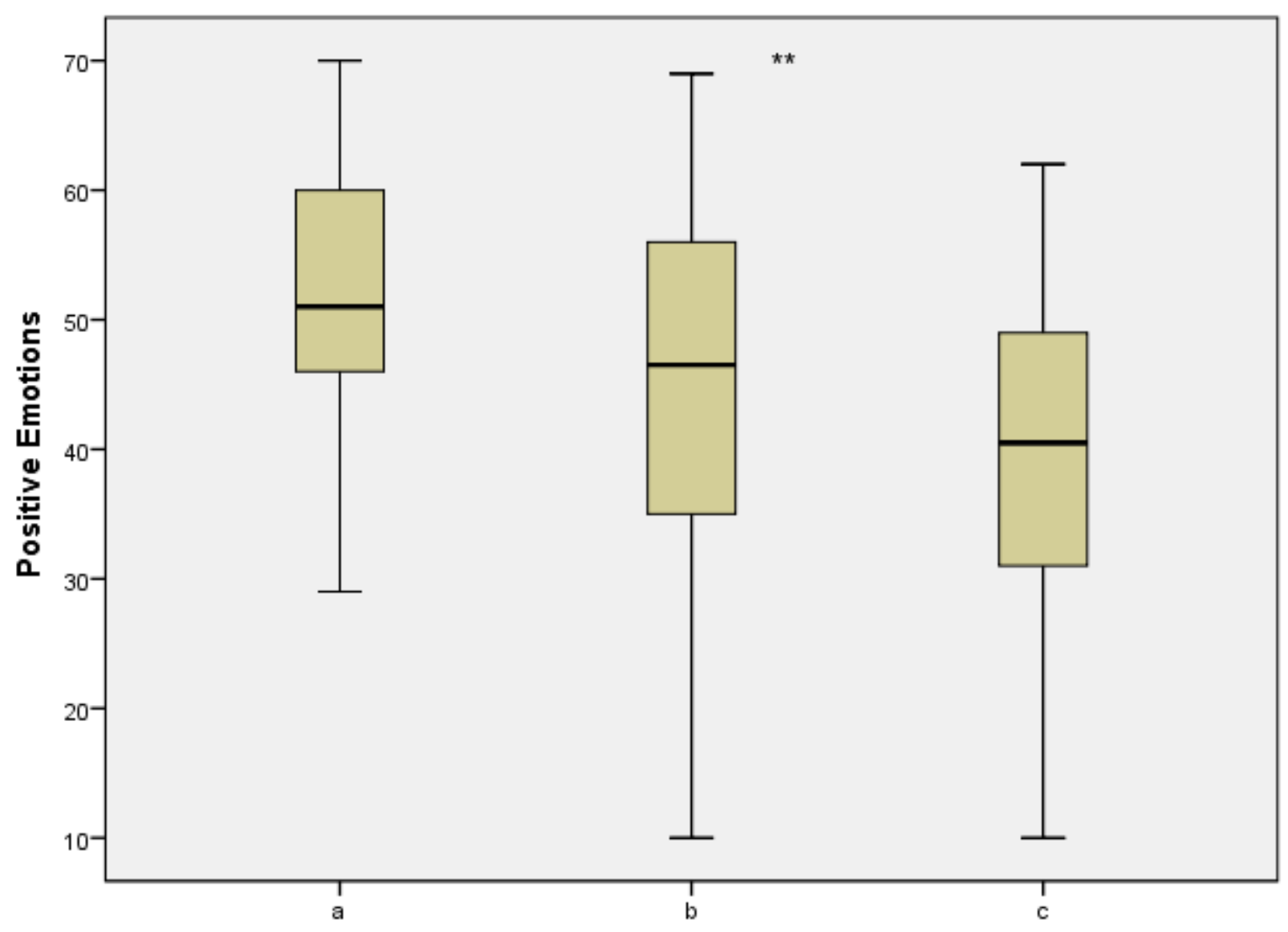

Patients groups according to the time of their assesment

Figure 2

Evolution of Positive Emotions level in function of Assessment Time

$\star \star p<0.01$

Group a: participants assessed the year before the first lockdown (March 17, 2020)

Group b: participants assessed from the beginning of the first lockdown to the end of second lockdown (March 17, 2020 - December 15, 2020)

Group c: participants assessed from the end of second lockdown - to the end of the lockdown measures in France (December 15, 2020 - June 20, 2021) 


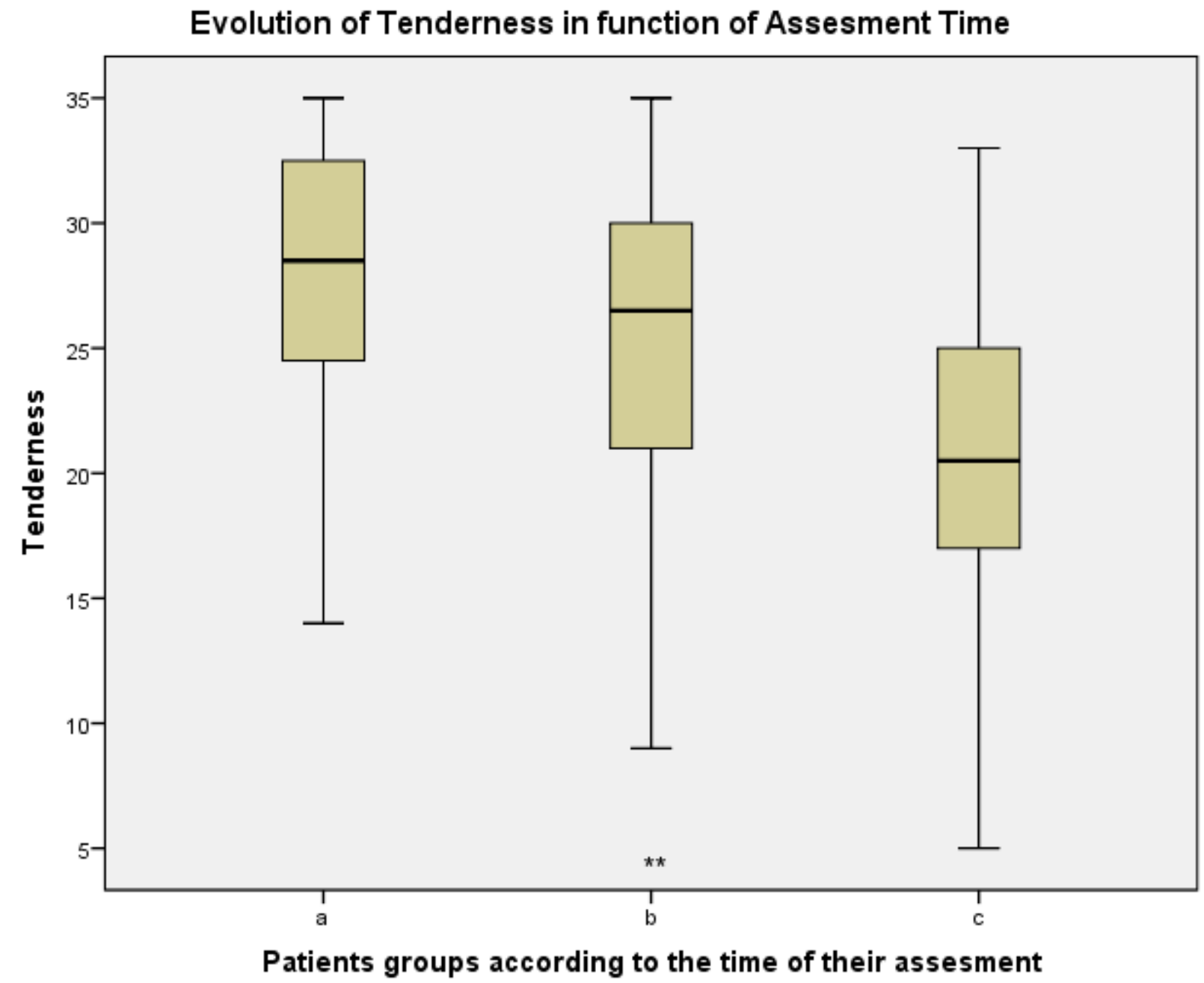

Figure 3

Evolution of Tenderness level in function of Assessment Time

$\star * p<0.01$

Group a: participants assessed the year before the first lockdown (March 17, 2020)

Group b: participants assessed from the beginning of the first lockdown to the end of second lockdown (March 17, 2020 - December 15, 2020)

Group c: participants assessed from the end of second lockdown -to the end of the lockdown measures in France (December 15, 2020 - June 20, 2021) 